\title{
LGRBs beaming features and SNIb/c connection in the light of the Second Swift BAT Catalog
}

\author{
Chadia Kanaan*1 \& J.A. de Freitas Pacheco ${ }^{1 \dagger}$ \\ ${ }^{1}$ University of Nice-Sophia Antipolis \& Observatoire de la Côte d'Azur \\ Laboratoire Lagrange, UMR 7293, BP 4229, F-06304, Nice Cedex 4, France \\ E-mail: chadia.kanaan@oca.eu, pacheco@oca.eu
}

\begin{abstract}
We present new estimate of the LGRB event rate in the local Universe. Adopting a Monte Carlo approach and assuming a Log-normal distributed emitted energy of the population of LGRBs, we derive a mock catalogue of simulated fluences for the five Swift energy bands. We than compare its corresponding distribution with the observed one by mean of best fit procedure. This gives a mean jet energy of $\log E_{\gamma}=49.48$ (in erg), where the observed redshift distribution is only well constrained after a moderate, linear evolution of the mean energy with redshift is been considered. Finally, we deduce the local formation rate to be $R_{G R B}=290 G p c^{3} y r^{1}$ representing less than $9 \%$ of the local formation rate of type Ib/c supernovae. The present results suggest that GRB progenitors, if following the cosmic star formation history, are very massive and potentially highly rotating stars.
\end{abstract}

Gamma-Ray Bursts 2012 Conference-GRB2012,

May 07-11, 2012

Munich, Germany

\footnotetext{
${ }^{*}$ Speaker.

$\dagger$ The author acknowledge travel support from the LOC of GRB2012
} 


\section{Introduction}

The estimate of the local formation rate of Long duration Gamma-Ray Bursts (LGRBs) $R_{G R B}$ give us a good hint about the underlying progenitor system. It allows us to explore the very wide grid of stellar evolutionary tracks possibly leading to typical GRB phenomena. The comparison of the local GRB rate with the other previously classified astrophysical stellar objects such as $\mathrm{SNIb} / \mathrm{c}$ could place constraints on the nature and features of the progenitor.

Practically, to calculate this quantity, we need to have a non-biased and complete sample of LGRB redshift and to assign a specific model for the distribution of volumetric cosmic mass rate with redshift. However, current observed sample is relatively small, highly biased and mission dependant: The measurement of the redshift distribution of LGRBs, exploding with a bolometric energy $E_{\gamma}$, is subject to a complex set of selection effects, occurring in the local environment of the burst (e.g., dust attenuation), along the line of sight, and/or related to the detector sensitivities and observability.

To overcome this problem, we propose, instead of dealing directly with the measured redshift distribution, to model the isotropic energy distribution of the prompt emission (not the luminosity as it is commonly done) and hence deduce the true redshift distribution that should be visible by the BAT/Swift detector. This is possible if LGRBs are standard energy reservoir, and thus can be modelled empirically within a single LGRBs population.

\section{Methods}

Investigating the "assumed standard" energy reservoir of Long duration $\gamma$-ray burst (LGRBs) has been a long-standing challenge for the GRB community. The major drawback is the limited size of the $E_{\gamma}$ sample proper to a single satellite. Nonetheless, the lack of $E_{\gamma}$ events has been quickly balanced by an important flow of fluences $f$ and durations $T_{90}$ data, especially in the light of the Swift satellite. This permits to constrain the model energy $\phi\left(E_{\gamma}, \mu_{0}(1+\alpha z), \sigma\right)$ through a secondary more frequent observable, being $f$ in the present work. Moreover, the true $E_{\gamma}$ distribution, as it is the case for redshift, is subject subsequently to geometrical and instrumental biases: A GRB is detected if the jet, with aperture $\theta_{J}$, is along the line of sight (LOS) and the detector is efficient enough to resolve the spectral shape of its hard X-ray emitted spectra, typically, a Band function $B\left(E_{v}, \alpha, \beta\right)$ [1] with peak energy $E_{p}$ and spectral indexes $\alpha$ and $\beta$.

To deal with such complexity, we have build a Monte Carlo code aiming to model log-normally the isotropic energy distribution. We assume $E_{\gamma}$ median distribution $\mu_{0}$ is evolving with redshift and that LGRBs form a single class population (XRF, XRR and CGRB combined) originating from direct collapse of massive stars. Their energetics are intrinsically rest-frame correlated, i.e, $E_{p_{0}}-E_{i s o}$, and thereby obey the Amati relation [6]. Our code closely follows the one developed by Lamb et al. (2005) [3] aiming to investigate the unified jet model. For each run, we simulate $z, E_{\gamma}, \theta_{J}, f(\varepsilon), T_{90}$, we construct a mock catalogue of detected events and we compare the corresponding $z$ and $f\left(\varepsilon_{i 1}, \varepsilon_{i 2}\right)$ distributions to the observed ones in the second Swift/BAT catalog [5]. Results are obtained by best fit procedure. For the sake of completeness, we summarize in Fig. 1 the main steps defining the sequence of calculations. 


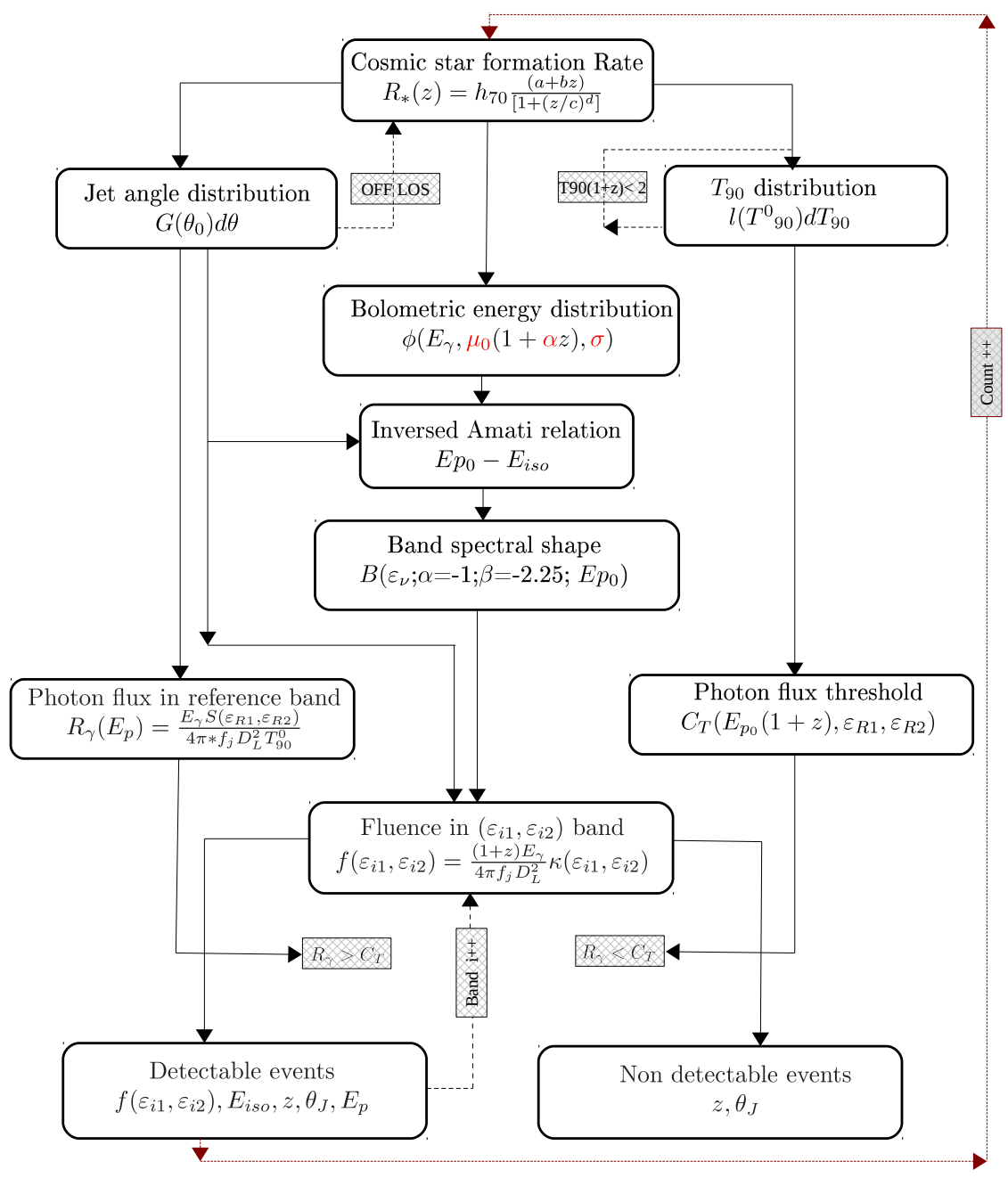

Figure 1: A schematic illustration of the simulation algorithm. It represents a single-run cycle for which one event is detected corresponding to single trial of the main variables being modelled, i.e $\mu_{0}, \alpha$ and $\sigma$.

\section{Results}

We performed simulations in both cases where GRB emits isotropically and under a simple jet structure. The redshift distributions predicted by the isotropic and the jet models without evolutionary effects underestimate the number of LGRBs occurring at $z>2$, in agreement with past investigations, requiring a moderate/strong evolution of the luminosity function. The fit of the redshift data (presently including 170 objects [2]) can be considerably improved if the jet energy distribution evolves in the sense that at high redshift bursts are more energetic. Such an evolution was estimated from our simulations such as the median $\mu_{0}$ of the log-normal describing the energy 


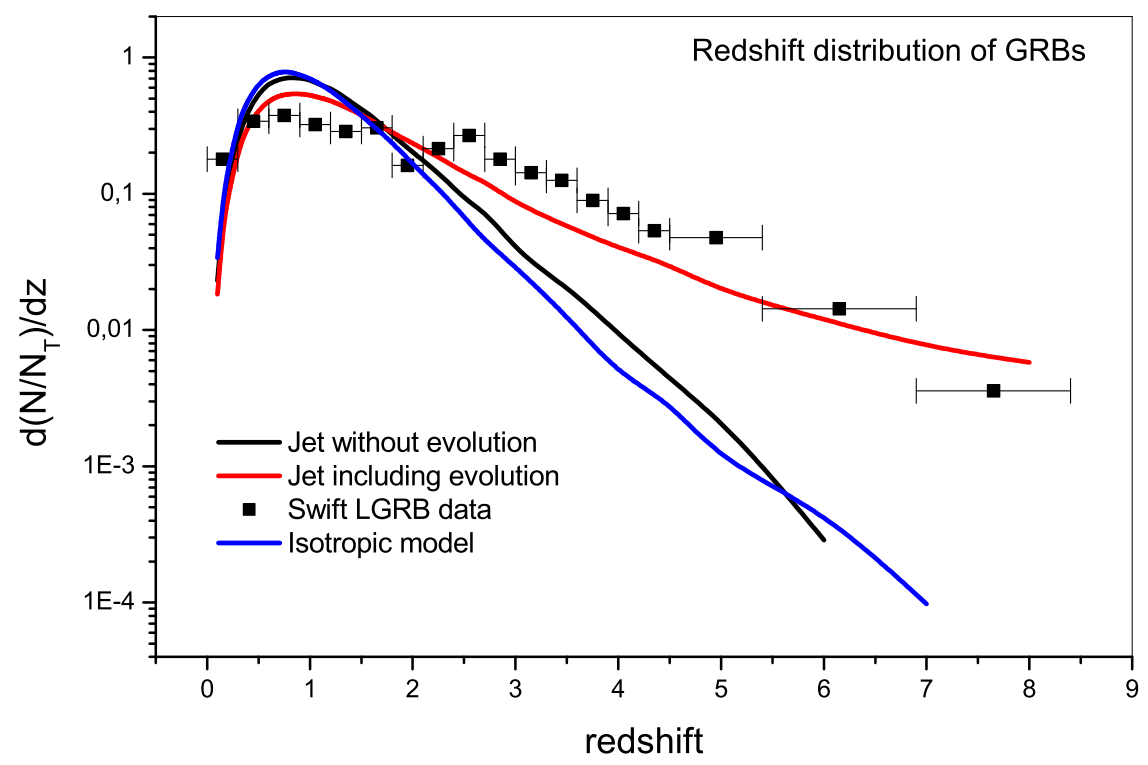

Figure 2: LGRB redshift distribution. Error bars indicate the bin width. The different curves correspond to distributions derived from the present simulations: isotropic (blue curve) and jet (black curve) models without evolution and jet model including evolution (red curve).

distribution varies as $E_{\gamma} \propto e^{0.5 z}$. For this model, the derived local formation is $R_{g r b}=290 \mathrm{Gpc}^{-3} y r^{-1}$ and the expected average jet energy is $3.0 \times 10^{49} \mathrm{erg}$, in nice agreement with the values derived by Lu et al. (2012) [4].

The aforementioned evolutionary jet model leads to a GRB fraction per unit mass of formed stars of $\lambda_{g r b}=2.8 \times 10^{-5} M_{\odot}^{-1}$. If the IMF does not evolve and if a Miller \& Scalo IMF is adopted, the derived value of $\lambda_{g r b}$ implies that the minimum mass to produce a LGRB is $90 M_{\odot}$, indicating that only very massive stars are associated to these events. Moreover, the ratio between LGRBs and SNIbc formation rates is in the range $0.01-0.09$, reinforcing the idea that stars less massive than the above limit may produce a SNIbc event but not necessarily a LGRB.

\section{References}

[1] D.L. Band, Postlaunch Analysis of Swift's Gamma-Ray Burst Detection Sensitivity, ApJ 644 (2006) 378 [astro-ph/0602267]

[2] N.R. Butler, J.S. Bloom and D. Poznanski, The Cosmic Rate, Luminosity Function, and Intrinsic Correlations of Long Gamma-Ray Bursts, ApJ 711 (2010) 495 [astro-ph/ 0910 . 3341 ]

[3] D.Q. Lamb, T.Q. Donaghy and C. Graziani, A Unified Jet Model of X-Ray Flashes, X-Ray-rich Gamma-Ray Bursts, and Gamma-Ray Bursts. I. Power-Law-shaped Universal and Top-Hat-shaped Variable Opening Angle Jet Models, ApJ 620 (2005) 355 [astro-ph/ 0312634 ] 
[4] R.-J. Lu , J.-J. Wei , S.-F. Qin and E.-W. Liang, Selection Effects on the Observed Redshift Dependence of Gamma-Ray Burst Jet Opening Angles, ApJ 745 (2012) 168 [astro-ph/1110.4943]

[5] T. Sakamoto, S.D. Barthelmy, W.H. Baumgartner et al., The Second Swift Burst Alert Telescope Gamma-Ray Burst Catalog, ApJS 195 (2011) 2 [astro-ph/1104 . 4689]

[6] L. Amati, F. Frontera and C. Guidorzi, Extremely energetic Fermi gamma-ray bursts obey spectral energy correlations, A\&A $\mathbf{5 0 8}$ (2009) 173 [astro-ph/0907.0384] 